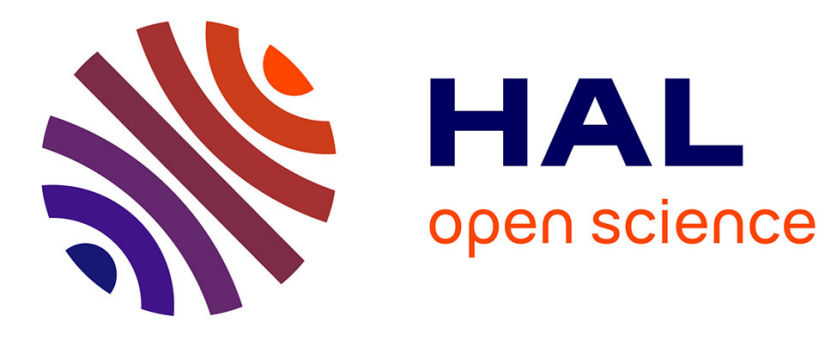

\title{
A Time Gap-Based Spacing Policy for Full-Range Car-Following
}

Carlos Flores, Vicente Milanés, Fawzi Nashashibi

\section{To cite this version:}

Carlos Flores, Vicente Milanés, Fawzi Nashashibi. A Time Gap-Based Spacing Policy for Full-Range Car-Following. Intelligent Transportation Systems Conference 2017, Oct 2017, Yokohama, Japan. hal-01634494

\section{HAL Id: hal-01634494 https://hal.inria.fr/hal-01634494}

Submitted on 14 Nov 2017

HAL is a multi-disciplinary open access archive for the deposit and dissemination of scientific research documents, whether they are published or not. The documents may come from teaching and research institutions in France or abroad, or from public or private research centers.
L'archive ouverte pluridisciplinaire HAL, est destinée au dépôt et à la diffusion de documents scientifiques de niveau recherche, publiés ou non, émanant des établissements d'enseignement et de recherche français ou étrangers, des laboratoires publics ou privés. 


\title{
A Time Gap-Based Spacing Policy for Full-Range Car-Following
}

\author{
Carlos Flores, Vicente Milanés and Fawzi Nashashibi.
}

\begin{abstract}
Car-following systems aim to improve safety and comfort whereas increasing traffic throughput. These techniques follow a spacing policy that determines how the egovehicle tracks its preceding one. This paper proposes a spacing policy to maximize the traffic throughput and reduce the intervehicle distances without losing safety and ensuring the string stability. A variable time gap policy is developed for low speeds, yielding the same dynamic response for high speeds and shorter spacing gaps. Simulations and real platforms' experiments are shown to validate the proposed approach.
\end{abstract}

\section{INTRODUCTION}

Urban and peri-urban traffic congestions are one of the main problems in the road transportation field. To further enhance the traffic throughput and road capacity, an effective solution is to optimize the way vehicles drive urban and peri-urban routes. This behavior can be modelled using a car-following strategy that defines how the inter-distances between the vehicles are kept. Such strategies reference the ideal spacing gap that the ego-vehicle should maintain in function of its state and its neighbour vehicles' states.

Human drivers perform car-following in common driving situations in a non-ideal manner. For this reason, several works have proposed to state a car-following strategy and an associated control law that acts over the longitudinal automation system of the ego-vehicle. In the literature, platooning maneuvers were the first techniques that applied carfollowing not only aiming to improve traffic capacity but also to save fuel if distances are short enough [1]. Even though it has provided good results in research projects [2], its proper implementation demands dedicated lanes and requires all string members to be constantly communicating with the leader to guarantee string stability. Nowadays, platooning has been mostly developed for short strings of heavy-duty trucks aiming fuel-efficient performances and exclusively for highway driving [3].

Other car-following techniques such as Adaptive Cruise Control (ACC) use ranging sensors to track the preceding vehicle and profit from longitudinal automation to maintain the desired spacing gap. It is an already commercial system and has been conceived to increase the driver comfort and smoothness when driving in highways by controlling throttle and brake pedals. Several works have studied the penetration rate impact of ACC systems in terms of traffic

Carlos Flores and Fawzi Nashashibi are with the Robotics and Intelligent Transportation Systems (RITS) Team, INRIA Paris, 2 Rue Simone Iff, 75012 France \{carlos.flores-pino, fawzi.nashashibi\}@inria.fr; Vicente Milanés is with the Research Department, Renault SAS, 1 Avenue de Golf, 78280 Guyancourt, France \{vicente.milanes@renault.com\}. capacity [4] and string stability [5]. Furthermore, if vehicleto-vehicle (V2V) communications are available, CooperativeACC (CACC) can be performed and shorter inter-distances can be maintained [6]. A literature review about diferent CACC implementations in highways can be found in [7].

These techniques apply a gap-regulation controller to command the longitudinal actuators and track the preceding vehicle. The control law aims to minimize the position error with respect to the inter-distance provided by a desired timegap policy. Numerous spacing policies have been proposed in the literature. They suggest different forward-vehicle tracking strategies that may consider a fixed distance, a time gap, the ego-velocity or the preceding vehicle speed. Each of them target different performance metrics such as comfort, safety, traffic capacity, traffic flow stability or string stability.

This paper proposes a novel spacing strategy that covers the full speed range, aiming to increment the traffic throughput, at the same time that the string stability is guaranteed. In terms of spacing, it is proposed a smooth transition between having a fixed distance in standstill and tracking a desired time gap for high speeds. This is carried out with the assignment of an initial and a target time gap for speed null and high speeds respectively, with a time gap adaptation process that sets the transition between both values. A fractional-based controller is proposed for the gap-regulation task since it provides robustness, damping properties and short time gaps among the string members without losing string stability.

The rest of this paper is structured as follows: Sec. II presents the motivation of this research. In Sec. III, the proposed spacing policy is described in details, while the employed control law for the preceding vehicle tracking is showed in Sec. IV. Validation results with both simulations and real platforms tests are described in Sec. V, demonstrating the benefits of applying the proposed full-range spacing policy. Some concluding remarks and possible future work are given in Sec. VI.

\section{WORK MOTIVATION}

The increase of traffic capacity has been demonstrated when implementing car-following capabilities over longitudinally automated vehicles. Below, a state-of-the-art review about the different car-following policies is carried out.

\section{A. Spacing policy review}

There is an extensive literature of car-following techniques where different spacing policies have been applied. As stated previously, each of them target different goals depending 
on the available sensors, the performed technique and the objective aimed.

1) Constant clearance was employed by the first platooning approaches [2], with the purpose of maintaining tightly-coupled strings. It proposes to keep short fixed distances that do not vary as the ego-speed varies. The reference distance is expressed as:

$$
d_{\text {ref }}=L ; L>0
$$

where $L$ is a fixed spacing that if short enough, fuel can be saved due to aerodynamic drag reduction. Consequently, it results ideal for close-formations of truck platooning. This policy requires low latency communication links with the platoon leader to guarantee string stability.

2) Constant time gap (CTG) is the most intuitive spacing policy [8]. It reflects human driver style as interdistance increases in a fixed rate when ego-vehicle speed increments. It proposes to keep a constant time gap $h$ multiplied by the ego-speed $v$ added to a fixed standstill distance $r$ :

$$
d_{\text {ref }}=r+h v ; r, h>0
$$

This time gap is measured from the front of the ego-vehicle to the rear-bumper of the preceding one. Such spacing policy has been widely employed in ACC/CACC techniques [8] and its effects on traffic have been studied extensively.

3) Safety distance spacing policy [9] states that the distance gap that should be maintained results from an estimation of the stopping distance required at the worst braking scenario (maximal acceleration of egovehicle and hard braking from the preceding). The proposed reference distance is determined as:

$$
d_{\text {ref }}=\beta_{1}+\beta_{2} v_{i}+\beta_{3}\left(v_{i}^{2}-v_{i-1}^{2}\right)
$$

where $\beta_{1}, \beta_{2}, \beta_{3}$ are constants that result from the stopping distance calculation and $v_{i}, v_{i-1}$ are the ego and preceding vehicles' speeds respectively. It can be noticed that in uniform driving scenarios-i.e. $\left(v_{i} \approx\right.$ $\left.v_{i-1}\right)$-the CTG spacing policy is obtained.

4) Constant safety factor (CSF) [3] constitutes a technique mostly considered for specific cases where the follower vehicle has less deceleration capabilities than the leading one, and even higher inter-distances must be kept to avoid collisions. It proposes to maintain a spacing gap that is a quadratic function of the speed:

$$
d_{\text {ref }}=\lambda_{1}+\lambda_{2} v+\lambda_{3} v^{2}
$$

5) Variable time headway and non-linear policies have gained attention in recent years due to their more flexible strategy. The former propose to modify the desired time gap in function of different purposes, while the latter suggest a non-linear function of the ego-speed among other variables. [10] proposes a time gap that varies within the range $h \in[0,1]$ depending on the relative velocity between the preceding and the ego-vehicle, achieving tightly coupled formations. Another technique [11] suggests to maintain a spacing proportional to the difference of the ego-speed and the string leader speed, aiming to increase the traffic density.

All these car-following techniques provide proper responses attending to different criteria but, regretfully, none of them provides string stable and safe responses in the full speed range whereas optimizing traffic throughput. Having this in mind, the aim of this work is to design a spacing policy that addresses the following objectives:

- Cover the full speed range, proposing a velocitydependant spacing strategy.

- Reduce/optimize the inter-distances between the vehicles to increase the traffic throughput maintaining safety.

- Ensure the string stability in the entire speed range.

- Assume that string information is only available from the preceding vehicle (either on-board sensors or V2V link)

\section{PROPOSED APPROACH}

In this section the proposed spacing policy is explained in detail for low and high speeds. Then, the design parameters as well as how to choose them properly for each techniquei.e. ACC or CACC-are explained.

\section{A. Time gap-based spacing policy}

The previously stated objectives are fulfilled by designing a full-range time gap-based spacing strategy that adapts the inter-vehicle distances properly in function of the ego-speed. The proposed car-following spacing policy is divided in urban and highway scenarios (i.e. low and high speeds). The former requires to keep a fixed standstill distance to provide stop-and-go capabilities, whereas the latter can remove such standstill distance, relaying on a constant time gap spacing (i.e. distances increases proportionally to a fixed time gap). Firstly, a CSF is proposed for low speeds to get a safe, continuous and smooth transition to high speeds, where the CTG strategy is applied seeking an uniform dynamic behavior and the desired car-following stability. The mentioned low speed time gap manipulation also optimizes the spacing yielding lower inter-distances for highway driving without losing the benefits and desired performance of CTG. For both cases, the policy design must guarantee that the referenced spacings produce safe car-following without possible frontend collision in case of preceding vehicle braking.

Focusing on the low speed scenario, the following requirements are stated to obtain the desired behavior: a fixed distance $r$ at standstill, a smooth and continuous transition to the target time gap $h_{\text {targ }}$ at the speed limit $V_{\text {lim }}$ between urban and highway scenarios and finally, ensured string stability and safety in the whole speed range. In other words:

$$
\begin{aligned}
& d_{r e f}(0)=r ; \\
& \frac{d_{r e f}(0)}{d v}=h_{\text {init }} ; \\
& \frac{d_{r e f}\left(V_{\text {lim }}\right)}{d v}=h_{\text {targ }} ;
\end{aligned}
$$


where $h_{\text {init }}$ is the time gap kept at standstill which defines the spacing evolution as the ego-speed increases. To satisfy the presented three requirements, a second order polynomial is suggested to describe the function $d_{r e f}(v)$ for low speeds. Consequently, a resulting spacing policy of the form:

$$
d_{r e f}(v)=\left\{\begin{array}{l}
\lambda_{1}+\lambda_{2} v+\lambda_{3} v^{2} ; 0 \leq v \leq V_{l i m} \\
h_{\text {targ }} v-c ; v \geq V_{\text {lim }}
\end{array}\right.
$$

is obtained and can be interpreted as a transition from CSF in low speeds to the CTG policy. The constant $c$ is an offset distance that ensures a continuous transition from low to high speeds and it also represents the distance saved with respect to a spacing given by $h_{\text {targ }} v$. After some manipulation, the parameters can be estimated to satisfy the requirements in Eq. 5. They are configured as follows:

$$
\begin{aligned}
& \lambda_{1}=r ; \\
& \lambda_{2}=h_{\text {init }} ; \\
& \lambda_{3}=\frac{\left(h_{\text {targ }}-h_{\text {init }}\right)}{2 V_{\text {lim }}} ;
\end{aligned}
$$

to produce a spacing strategy that satisfies the previously stated objectives. After determining the polynomial constants, the selection of the design parameters $\left(V_{\text {lim }}, h_{\text {targ }}, h_{\text {init }}, r\right)$ is carried out.

\section{B. Design parameters}

As explained before, the proposed strategy is divided in low and high speeds, where $V_{\text {lim }}$ sets the limit between both. Consequently, such parameter is selected to be the speed limit in urban areas and set the boundary between both scenarios.

Firstly, for ACC it is proposed to set $h_{\text {targ }}$ as a time gap chosen by the driver to be maintained in highways (usually between 1 and 1.5 seconds), whereas for CACC a lower time gap is set to increase the traffic throughput and also considering human factors guidelines for CACC driving in highways [12].

Analysing the low speed scenario (Eq. 6), the CSF policy can be translated as a time gap-based strategy to study the dynamic behavior through an equivalent time gap $h_{e q}(v)$ that increments as the speed increases. It results as a smooth function of the ego-speed, given that it can be expressed as the reference inter-distance derivative with respect to the ego-speed. Then, such value results for the full speed range as:

$$
h_{e q}(v)=\left\{\begin{array}{l}
h_{\text {init }}+\frac{\left(h_{\text {targ }}-h_{\text {init }}\right)}{V_{\text {lim }}} v ; 0 \leq v \leq V_{\text {lim }} \\
h_{\text {targ }} ; v \geq V_{\text {lim }}
\end{array}\right.
$$

Although lower time gaps enhance traffic capacity, they also yield less stable behavior and a more demanding gapregulation task is then required. Due to this fact, the minimum equivalent time gap $\left(h_{\text {init }}\right)$ is desired to be the lowest time gap that the employed control structure can afford ensuring string stability.

The parameter $r$ is chosen taking into consideration that the minimum inter-distance should be safe in the entire

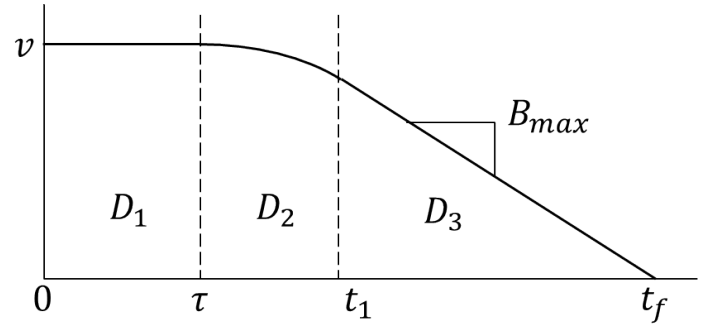

Fig. 1. Illustration of the distance that covers the ego-vehicle when a stopping maneuver is performed by the preceding one

range in case a braking maneuver is performed by the preceding car. The desired inter-distance must be higher than the minimum critical distance $d_{r e f, c r i t}(v)$ required to avoid a possible collision at any speed. The calculation of such distance is done assuming the vehicles are driving uniformly-i.e. no oscillations or high speed variations-as is the case when performing car-following. In such situation, the available distance $d_{a v}(v)$ that the ego-vehicle has to stop is defined by:

$$
d_{a v}(v)=d_{r e f}(v)+\frac{v^{2}}{2 B_{\max }} ;
$$

which is composed by the referenced spacing $d_{\text {ref }}(v)$ and the distance that covers the preceding vehicle when performing a braking with deceleration $B_{\max }$ from speed $v$. On the other hand, the spacing that requires the ego-vehicle to fully stop is described in Fig. 1 and is composed by the addition of three distances $D_{1}, D_{2}, D_{3}$ that are defined as follows:

$$
\begin{gathered}
D_{1}=\tau v ; \\
D_{2}=\frac{B_{\max } v}{J_{\max }}-\frac{B_{\max }^{3}}{6 J_{\max }^{2}} ; \\
D_{3}=\frac{v^{2}}{2 B_{\max }}-\frac{B_{\max } v}{2 J_{\max }}+\frac{B_{\max }^{3}}{8 J_{\max }^{2}} ;
\end{gathered}
$$

where $B_{\max }$ and $J_{\max }$ are the maximum braking deceleration and jerk respectively. These values are chosen assuming comfortable [13] car-following driving. The distance $D_{1}$ results from the time $\tau$ that represents actuator response's delay, whereas $D_{2}$ results from the travelled distance while reaching maximal deceleration $B_{\max }$ with a rate of $J_{\max }$. Finally, $D_{3}$ is the covered distance maintaining the mentioned deceleration rate until the car stops.

To ensure safety in a possible braking situation, and considering Eq. 9 with Eq. 10-12, the following condition must be fulfilled:

$$
d_{\text {ref }}(v) \geq d_{\text {ref,crit }}(v)=D_{1}+D_{2}+D_{3}-\frac{v^{2}}{2 B_{\max }}
$$

which constitutes the critical reference inter-distance that can be maintained. For the homogeneous case it results of the form:

$$
d_{\text {ref,crit }}(v)=\left(\tau+\frac{B_{\max }}{2 J_{\max }}\right) v-\frac{B_{\max }^{3}}{24 J_{\max }^{2}} ;
$$




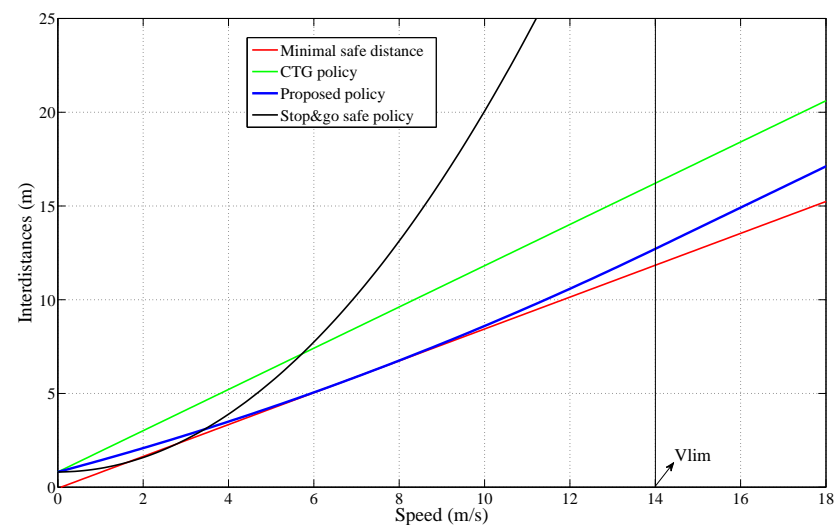

Fig. 2. Resulting full-range spacing policy (blue line), CTG policy (green line), safe longitudinal distance for ACC and stop\&go scenarios (black line) and the minimal spacing to keep (red line)

and defines the safe region in the spacing vs. speed plane, where the referenced distance has to be maintained above. Transition from low to high speeds is obtained at $V_{\text {lim }}$ combining Eq. 6 and 14. Subsequently, this condition is satisfied when:

$$
r=\frac{V_{\text {lim }}\left(h_{\min }-\tau-\frac{B_{\max }}{2 J_{\max }}\right)^{2}}{2\left(h_{\text {targ }}-h_{\text {init }}\right)}-\frac{B_{\max }^{3}}{24 J_{\max }^{2}} ;
$$

Fig. 2 shows the comparison between the resulting interdistance function and other state-of-the-art policies. Black line represents the car-following approach proposed in [14]. One can appreciate how its performance in terms of traffic optimization is significantly degraded when speed increases. Dotted red line represents the minimum safety distance to avoid a rear-end crash as calculated according to Eq. 14 . Green line depicts the so-called constant time gap policy. Finally, blue line shows the proposed spacing policy. Comparing the latter with CTG, it can be appreciated that the proposed approach modifies the curve slope in low speeds to optimize the separation between vehicles. This not only improves the traffic capacity if implemented in large scale strings but also ensures the same desired dynamic response for high speeds.

\section{Proposed Control LaW}

From the control perspective, lower time gaps directly affect string stability. The control structure designed for the gap-regulation task must guarantee the string stability for all the equivalent time gaps in the full speed range. The platform employed for the control development and further approach validation is the 4-wheel electrical vehicle prototype Cybercar [15]. A second-order transfer function is set to describe its dynamics, which is of the form:

$$
G p(s)=\frac{v_{\text {real }}(s)}{v_{\text {ref }}(s)}=\frac{1}{1+2 \xi T_{w_{n}} s+T_{w_{n}}^{2} s^{2}}
$$

where $\xi$ is the damping factor and $T_{w_{n}}$ represents the model natural period, which correspond to 0.385 and 0.334 respectively for the Cybercar. The model $G p(s)$ is considered for the control structure and further used in the controller design.

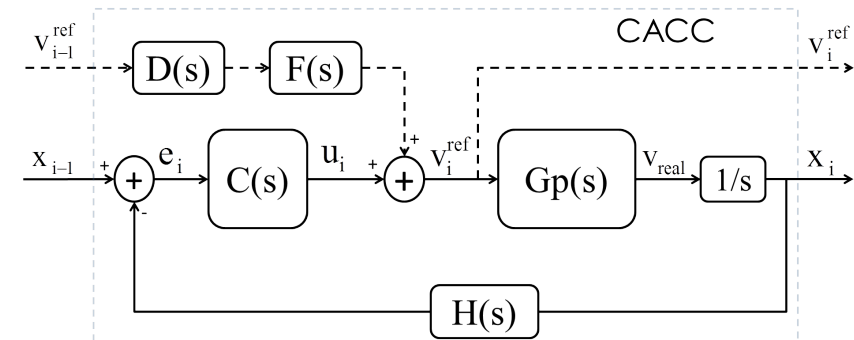

Fig. 3. CACC string stability control structure

\section{A. Control structures}

To correctly perform a gap-regulation task, a control structure is employed that considers the developed spacing policy in the loop. A feed-forward structure is used [16], which expression is described in the left term of the condition:

$$
\left\|\frac{X_{i}(s)}{X_{i-1}(s)}\right\|_{\infty}=\left\|\frac{D(s) F(s)+G p(s) C(s)}{1+G p(s) C(s) H(s)}\right\|_{\infty} \leq 1 ; i \geq 2
$$

which has to be fulfilled to guarantee the system string stability. $D(s)$ models the communication delays in the frequency domain as $e^{-\theta s} ; \theta>0 . H(s)$ is composed by the proposed spacing policy, which is of the form $1+h_{e q}(v) s$ where $h_{e q}$ is given by Eq. 8. The block $F(s)$ is a first order filter of the form: $F(s)=\frac{1}{H(s)}$ (see Fig. 3) which not only smooths the received feed-forward signal but also guarantees string stability for every time gap bigger than zero if no communication delays are present [17]. The block $G p(s)$ is the function that results from the vehicle identification process (see Eq. 16). Finally, the block $C(s)$ represents the controller in charge of the gap-regulation task, which output is added to the feed-forward term. If communication links are not available, the control structure can be adapted to perform ACC instead, by substituting the feed-forward term by the real speed $v_{\text {real }}$. By doing so, one obtains an inner feedback loop which receives the controller output as unique input.

\section{B. Controller design}

Fractional-order calculus is considered to profit from its accurate frequency response design and capabilities. In the literature, these controllers have been employed for gap-regulation techniques [18], [19] showing good results. For this work, the selected controller is a fractional-order Proportional-Derivative (FOPD) controller to benefit from its damping and stabilizing properties. It can be defined as:

$$
C(s)=\frac{E(s)}{U(s)}=K p+K d \cdot s^{\alpha} ;
$$

having $K p$ as proportional gain, $K d$ as derivative gain and $\alpha$ as the non-integer derivation order that exists in the range of $\alpha \in(0,2)$.

For the controller parameters tuning, the method proposed in [19] is followed to tune the FOPD. Such type of controller allows to fulfil three design requirements. As in the 


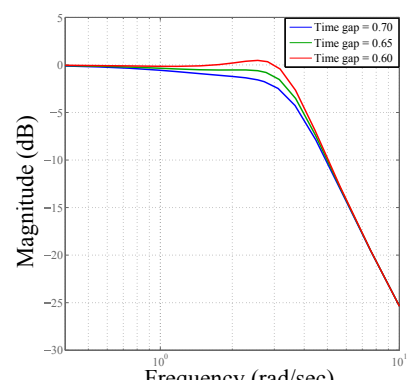

Frequency $(\mathrm{rad} / \mathrm{sec})$

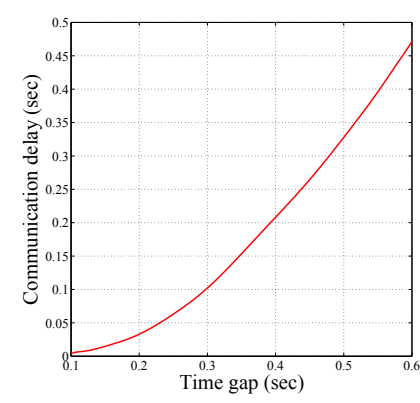

Fig. 4. String stability limits of the proposed ACC and CACC control structures

referenced method, it is desired to increase the open-loop response bandwidth, improve the phase margin and guarantee robustness against plant variations-i.e. phase flatness in the gaincross frequency.

Regarding the string stability, it is known that the lower the time gap targeted the closer the system gets to its string stability limit. Consequently, to ensure the string stability in the full speed range, the fulfilment of such condition has to be demonstrated for the lowest time gap suggested by the policy-i.e. $\min \left(\partial d_{\text {ref }} / \partial v\right)=h_{\text {init }}$. After studying the control structure stability limit, Fig. 4 permits to choose the $h_{\text {init }}$ that corresponds to ACC and CACC respectively.

\section{VALIDATION}

To demonstrate the correct performance and the benefits of the proposed spacing policy, simulations and a comparison with different state-of-the-art techniques are carried out. Then, real tests on a Cybercar are shown with the described control structures and spacing strategy.

\section{A. Simulations}

A two-string vehicle is used to evaluate the performance. Three car-following policies are compared through the ACC technique: the proposed full-range spacing policy, CTG and the safety distance policy [9]. The vehicles' speeds and spacings are evaluated in the Fig. 5 in the upper and lower plot respectively. The employed standstill distance is set to 0.35 meters and the target time gap is 1.1 second.

It can be appreciated that in terms of speed the proposed approach and the CTG policies are similar, which is due to the fact that both are based on time gaps. On the other hand, the safe ACC policy tracks the preceding vehicle in a more conservative manner. This can be noticed in the interdistances plot, which shows that this technique references higher spacings as well as slower speed transitions. In the same plot, the proposed full-range and the CTG policies perform similarly in low speeds in terms of spacing, but as the speed increases one can distinguish that the former strategy suggests to maintain lower inter-distances than the CTG. Regarding the string stability, it is clear that the car-following maneuver is executed in a stable way and consequently the amount of vehicles that composes the string can be increased without leading to an undesired behavior.
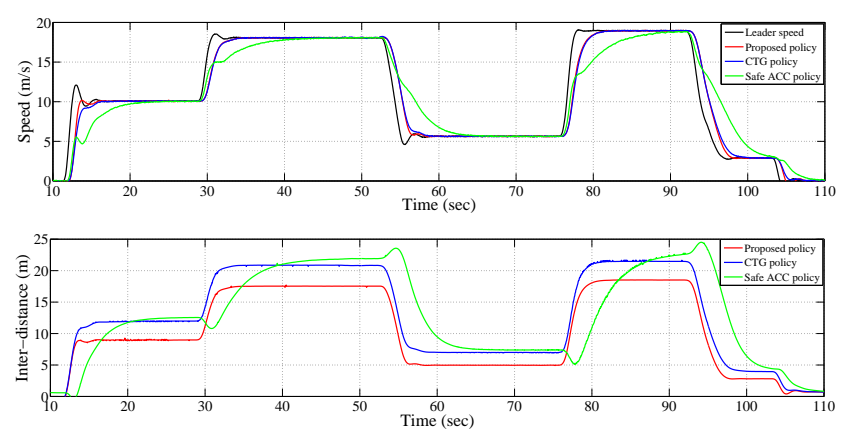

Fig. 5. Speed comparison between vehicles employing the three different policies
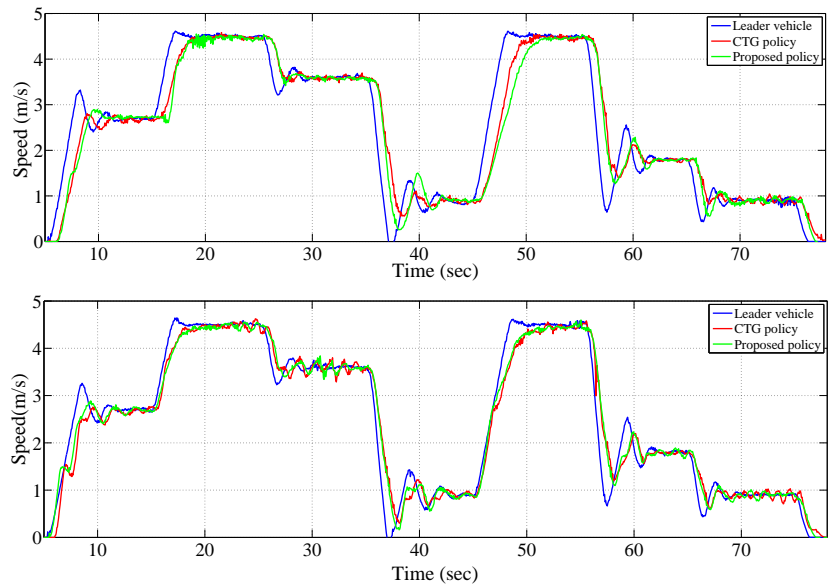

Fig. 6. Leader vehicle (blue line), CTG and full-range policies followers' (red and green lines respectively) speeds using ACC (upper plot) and CACC (lower plot)

\section{B. Real platforms tests}

The proposed algorithm was also tested on the experimental platform at INRIA test tracks and compared against the CTG policy. The first vehicle follows a speed profile with several speed changes to see the inter-distance evolution as well as the tracked time gap that outputs the algorithm for both cases. Due to the fact that the Cybercar is not able to drive at speeds higher than $5 \mathrm{~m} / \mathrm{s}$, the tests are scaled to adjust the speed range of such platform. The speed boundary $V_{\text {lim }}$ between low and high speeds is configured as $4 \mathrm{~m} / \mathrm{s}$. The tests are carried out using firstly ACC and afterwards the same speed profile is performed using CACC.

Fig. 6 depicts the speed profile followed by the leader vehicle and how each of the following cars are tracking it both for ACC and CACC. As expected, the CACC follower reacts in a faster way towards leader speed changes than the ACC one, which is produced by the lower time gaps that are targeted and the employed communication links.

In the upper plot of Fig. 7, a comparison between the spacing evolution when employing CTG and the proposed strategy is depicted. At low speeds the behavior results similar in terms of spacing, but as the speed increases to $V_{\text {lim }}=4 \mathrm{~m} / \mathrm{s}$ the inter-distance optimization targeted by 

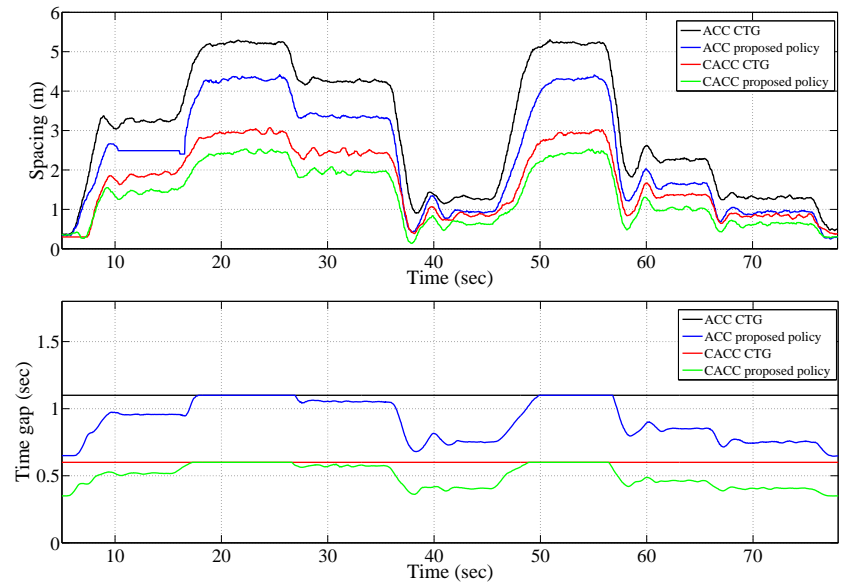

Fig. 7. ACC and CACC performances described through the spacing (upper plot) and the equivalent time gaps targeted (lower plot)

the proposed approach is demonstrated as it stays lower than the CTG strategy. Such performance is produced by the adaptation of the equivalent time gap from the minimum ( $0.65 \mathrm{sec}$ for ACC and $0.35 \mathrm{sec}$ for CACC) to the target time gap (1.1 sec for ACC and $0.6 \mathrm{sec}$ for CACC) as the speed increments (see lower plot of Fig. 7). It is also possible to distinguish the transition moment between low speeds zone and high speeds, since the time gap stays at the targeted value as expressed in Eq. 8 and the inter-distance difference between CTG and the full-range policy stays constant.

\section{CONCLUSIONS}

This works presents a time gap-based car-following spacing policy that covers the full speed range for ACC and CACC systems. A review of the state-of-the-art spacing strategies is carried out with an analysis of their properties. For the gap-regulation task, a fractional-order controller has been proposed and employed in the validation stage. Simulations taking into consideration other strategies are carried out to validate the approach and demonstrate its benefits. Real platforms' experiments validate the proposed carfollowing policy inter-distance optimization, keeping string stable responses even employing information only from the preceding vehicle.

\section{ACKNOWLEDGMENTS}

Authors express their gratitude to the french project VALET and the RITS Team for its support in the development of this work.

\section{REFERENCES}

[1] F. Browand, J. McArthur, and C. Radovich, "Fuel saving achieved in the field test of two tandem trucks," California Partners for Advanced Transit and Highways (PATH), 2004.

[2] R. Rajamani, H.-S. Tan, B. K. Law, and W.-B. Zhang, "Demonstration of integrated longitudinal and lateral control for the operation of automated vehicles in platoons," IEEE Transactions on Control Systems Technology, vol. 8, no. 4, pp. 695-708, 2000.
[3] J. B. Michael, D. N. Godbole, J. Lygeros, and R. Sengupta, "Capacity analysis of traffic flow over a single-lane automated highway system," Journal of Intelligent Transportation System, vol. 4, no. 1-2, pp. 4980, 1998.

[4] X. Zou and D. Levinson, "Evaluation of impacts of adaptive cruise control on mixed traffic flow," in Traffic And Transportation Studies (2002), 2002, pp. 762-769.

[5] L. Xiao and F. Gao, "Practical string stability of platoon of adaptive cruise control vehicles," IEEE Transactions on Intelligent Transportation Systems, vol. 12, no. 4, pp. 1184-1194, Dec. 2011.

[6] S. E. Shladover, C. Nowakowski, X.-Y. Lu, and R. Ferlis, "Cooperative adaptive cruise control: Definitions and operating concepts," Transportation Research Record: Journal of the Transportation Research Board, no. 2489, pp. 145-152, 2015.

[7] K. C. Dey, L. Yan, X. Wang, Y. Wang, H. Shen, M. Chowdhury, L. Yu, C. Qiu, and V. Soundararaj, "A review of communication, driver characteristics, and controls aspects of cooperative adaptive cruise control (cacc)," IEEE Transactions on Intelligent Transportation Systems, vol. 17, no. 2, pp. 491-509, 2016.

[8] D. Swaroop and K. R. Rajagopal, "A review of constant time headway policy for automatic vehicle following," in Proc. (Cat. No.01TH8585) 2001 IEEE Intelligent Transportation Systems ITSC 2001, 2001, pp. 65-69.

[9] P. A. Ioannou and C.-C. Chien, "Autonomous intelligent cruise control," IEEE Transactions on Vehicular technology, vol. 42, no. 4, pp. 657-672, 1993.

[10] D. Yanakiev and I. Kanellakopoulos, "Nonlinear spacing policies for automated heavy-duty vehicles," IEEE Transactions on Vehicular Technology, vol. 47, no. 4, pp. 1365-1377, 1998.

[11] A. Ali, G. Garcia, and P. Martinet, "Minimizing the inter-vehicle distances of the time headway policy for platoon control on highways." in ICINCO (2), 2013, pp. 417-424.

[12] C. Nowakowski, S. E. Shladover, D. Cody, F. Bu, J. O'Connell, J. Spring, S. Dickey, and D. Nelson, Cooperative adaptive cruise control: Testing drivers' choices of following distances. California PATH Program, Institute of Transportation Studies, University of California at Berkeley, 2010.

[13] ISO, Mechanical Vibration and Shock: Evaluation of Human Exposure to Whole-body Vibration. Part 1, General Requirements: International Standard ISO 2631-1: 1997 (E). ISO, 1997.

[14] J.-J. Martinez and C. Canudas-de Wit, "A safe longitudinal control for adaptive cruise control and stop-and-go scenarios," IEEE Transactions on control systems technology, vol. 15, no. 2, pp. 246-258, 2007.

[15] J. E. Naranjo, L. Bouraoui, R. Garcia, M. Parent, and M. . Sotelo, "Interoperable control architecture for cybercars and dual-mode cars," IEEE Transactions on Intelligent Transportation Systems, vol. 10, no. 1, pp. 146-154, Mar. 2009.

[16] J. Ploeg, B. T. M. Scheepers, E. van Nunen, N. van de Wouw, and H. Nijmeijer, "Design and experimental evaluation of cooperative adaptive cruise control," in Proc. 14th Int. IEEE Conf. Intelligent Transportation Systems (ITSC), Oct. 2011, pp. 260-265.

[17] G. Naus, R. Vugts, J. Ploeg, R. v. d. Molengraft, and M. Steinbuch, "Cooperative adaptive cruise control, design and experiments," in Proc. American Control Conf, Jun. 2010, pp. 6145-6150.

[18] S. H. Hosseinnia, I. Tejado, V. Milanés, J. Villagrá, and B. M. Vinagre, "Experimental application of hybrid fractional-order adaptive cruise control at low speed," IEEE Transactions on Control Systems Technology, vol. 22, no. 6, pp. 2329-2336, Nov. 2014.

[19] C. Flores, V. Milanés, and F. Nashashibi, "Using fractional calculus for cooperative car-following control," in IEEE 19th International Conference on Intelligent Transportation Systems (ITSC), 2016. IEEE, 2016, pp. 907-912. 